\title{
Convergence Paralysis as a Manifestation of Polyarteritis Nodosa
}

\author{
Jessica Wylie, Craig Campbell, Janet Pope, Jonathan Akikusa, Ronald M. Laxer, Dave Nicolle
}

Can. J. Neurol. Sci. 2006; 33: 423-425

Polyarteritis nodosa (PAN) is a rare, systemic necrotizing vasculitis of medium-sized arteries. The American College of Rheumatology criteria for the diagnosis of PAN includes at least three of: 1 . weight loss $>4 \mathrm{~kg}, 2$. livedo reticularis, 3. testicular pain or tenderness, 4. myalgias, weakness or leg tenderness, 5. mono-or polyneuropathy, 6. diastolic hypertension, 7. elevated blood creatinine or urea, 8 . hepatitis B antigen or antibody in the serum, 9. aneurysms or occlusions of visceral arteries or 10. granulocytes on small or medium sized artery biopsy. ${ }^{1}$ Common sites of involvement include skin, joints, kidneys, gastrointestinal tract and peripheral nerves. Central nervous system involvement has been reported in up to $40 \%$ of cases; the usual manifestations are encephalopathy, focal deficits and seizures. ${ }^{2}$ Descriptions of PAN in the pediatric literature has been reported in fewer than 250 children. ${ }^{3}$

A previously well 15 year old female presented with a two week history of intermittent headache associated with one week of episodic vomiting and vertigo. She also complained of diplopia (horizontal and diagonal), falling to the right side while walking and a fullness in her right ear that made her unable to hear. Further history revealed that she had a "pins and needles" feeling in her left hand and right foot that had been present for three months. She complained of bilateral leg pain. As well, she described a lacy rash on her hands and legs that appeared when exposed to the cold and changed colour from red to purple. The patient also described a six week history of intermittent "stabbing" epigastric pain and a 25 pound weight loss. Her only medication was minocycline $250 \mathrm{mg}$ once daily, which she had been taking for two years to treat acne. Family history revealed that her maternal grandmother had both systemic erythematous lupus and rheumatoid arthritis.

On physical examination, she was afebrile. Her blood pressure was elevated at 138/94, the remainder of her vital signs were normal. Her neurologic exam revealed bilateral exotropia; however she was able to fix with both eyes. Extraocular movements were full when each eye was tested independently. She had convergence failure with marked limitation of adduction in the right eye. Her left pupil was mildly dilated but both pupils were reactive to light. Fundoscopy was normal. She did not have nystagmus. There was hearing loss in the right ear. Her Romberg test was positive and her gait also demonstrated falling to the right side. She had muscle wasting, fasciculations, and a sensory deficit of the hypothenar eminence, fifth digit and ulnar side of the fourth digit of the left hand and an isolated sensory deficit to light touch on the medial plantar aspect of her right foot. The remainder of both her motor and sensory exams were normal.
Livedo reticularis was present on her hands and legs, no bruits were heard. The rest of her general examination was normal.

A CT scan of the head was normal. An MRI of the head showed a 2-3 $\mathrm{mm}$ focus of increased signal within the left paramedian anterior midbrain on DWI and T2 weighted images thought to represent an acute perforator territory infarct (see Figure 1 and 2). Other MRI sequences including an MRA did not demonstrate an abnormality. An MRA of her abdominal vessels was normal.

A lumbar puncture was performed with an opening pressure of $12 \mathrm{mmHg}$, an elevated CSF protein of $500 \mathrm{mg} / \mathrm{L}$ and absent oligoclonal bands. She had mild elevations of erythrocyte sedimentation rate (ESR, $29 \mathrm{~mm} / \mathrm{h}$ ), C-reactive protein (CRP, 21 $\mathrm{mg} / \mathrm{L})$ and platelets $\left(474 \times 10^{9} / \mathrm{L}\right)$. Her ANA was positive at 1:640 with a mixed atypical speckle pattern. p-ANCA and cANCA were normal. Quantitative Epstein-Bar virus and Cytomegalovirus studies were negative. Urinalysis was normal. Other studies revealed electrolytes, lactate, calcium, glucose, hemoglobin, white blood cell count, INR, PTT, lupus anticoagulant, anti DNA, anti ENA, rheumatoid factor, complement, protein $\mathrm{C}$ and $\mathrm{S}$, and antithrombin III were all normal.

Left ulnar motor nerve conduction studies showed a normal distal latency $(4.2 \mathrm{~ms}$ at $7 \mathrm{~cm})$, a reduced amplitude in FDI (3.2 $\mathrm{mV}$ ) and ADM (2.6mV), and normal conduction velocities in distal and across elbow segments $(>46.2 \mathrm{~m} / \mathrm{s})$. A left deep peroneal study to extensor digitorum brevis showed reduced amplitude $(0.4 \mathrm{mV})$ and reduced conduction velocity in the lower leg $(35.9 \mathrm{~m} / \mathrm{s})$ and across the fibular head $(28 \mathrm{~m} / \mathrm{s})$. Sensory responses from sural and superficial peroneal nerves were absent bilaterally. These nerve conduction studies demonstrated an ulnar neuropathy arising proximal to the left elbow, a right peroneal neuropathy and bilateral sensory

From the Department of Pediatrics (JW, CC), Rheumatology (JP), Ophthalmology (DN), London Health Sciences Centre, University of Western Ontario, London, ON; Rheumatology, Pediatrics and Medicine, (JA, RML), Hospital for Sick Children, University of Toronto, Toronto, ON, Canada.

Received February 8, 2006. AcCePted in final form August 18, 2006. Reprint requests to: Craig Campbell, Section of Pediatric Neurology, Children's Hospital of Western Ontario, 800 Commissioners Rd. E., London, Ontario N6A 2E3, Canada. 


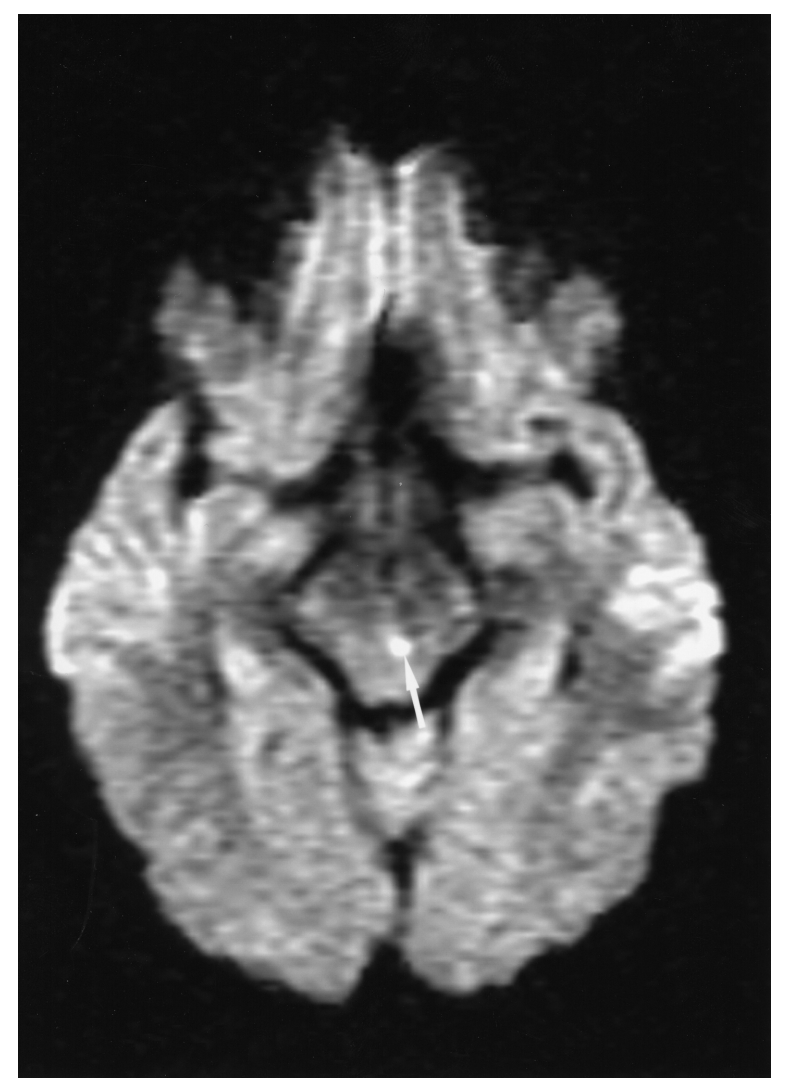

Figure 1. The MRI demonstrating infarct in the left paramedian midbrain. Axial diffusion weighted scan.

neuropathies of her feet. The following motor nerve conduction studies were normal: left median, right ulnar, left common peroneal, left tibial. The following sensory nerve conduction studies were normal: left radial, left median, right ulnar, bilateral medial antebrachial cutaneous. Electromyography showed spontaneous activity and large amplitude motor responses from her left FCU, left FDI muscle and left EDB. An audiogram revealed moderate sensorineural hearing loss in the right ear. Acoustic impedance testing showed normal middle ear function bilaterally. Brainstem evoked potential audiometry was within normal limits in the left ear. In the right ear, Wave I was not present, Wave III was delayed but there was normal Wave V latency, and a normal Wave III-V interval was present. This audiology assessment suggested a cochlear nerve lesion and a lack of significant brainstem auditory pathway involvement. Her ECG was normal.

On the basis of unexplained weight loss, myalgia, mononeuritis multiplex, diastolic hypertension and the presence of livedo reticularis a diagnosis of PAN was made and she was started on prednisone, aspirin, amlodipine and later azathioprine. Neuropathic pain improved with gabapentin. At follow-up two years later the child is in remission on azathioprine and is asymptomatic aside from right ear hearing deficit and hypertension controlled by medication.

\section{Discussion}

The patient described has a series of neurological deficits including 1. convergence paralysis, 2. partial left oculomotor nerve palsy, 3. ataxia, 4. right ear hearing impairment, 5. left proximal ulnar neuropathy, and 6. motor and sensory polyneuropathy in the lower extremities. The convergence paralysis, dilated left pupil, and disequilibrium are secondary to a midbrain stroke. The hearing deficit is related to a cochlear nerve lesion rather than brainstem pathology. The ataxia is related either to peripheral vestibular or cerebellar output (i.e. red nucleus) dysfunction. The peripheral neuropathy is consistent with a mononeuritis multiplex. In addition to these ischemic vascular pathologies, her livedo reticularis, hypertension, myalgias and weight loss make the diagnosis of PAN applicable on clinical grounds. Given all these features it was felt a nerve or skin biopsy was not required for diagnosis.

A stroke in the midline of the midbrain due to an inflammatory lesion of the paramedian penetrating arteries of the basilar artery is responsible for our patients' ocular findings, as infarction in this area can affect the third nerve root fibers and the red nucleus. ${ }^{4}$ Convergence relies on simultaneous activation of adductor nuclei of third cranial nerve and convergence fibres which must exist in the midline of the very complex oculomotor nucleus. Convergence paralysis is characterized by diplopia at near fixation yet normal adduction otherwise and is typically caused by a midbrain lesion. ${ }^{5}$

In adult studies of PAN, central nervous system (CNS) manifestations are found in $10 \%$ to $40 \%$ of patients. $2,6-8$ The clinical presentation is variable and may include the following symptoms and signs: headache, blurred vision, tinnitus, encephalopathy, hemiparesis, cranial nerve palsies, seizures, and strokes. ${ }^{6}$ Cranial nerve palsies are present in less than $2 \%$ of patients, with the III, IV, VI, VII and VIII being affected most often. ${ }^{9}$ Peripheral neuropathy is present in $50-75 \%$ of cases of PAN and tends to occur earlier than CNS manifestations. ${ }^{9-11}$ Mononeuritis multiplex is the most frequent and earliest neurological manifestation of PAN occurring in 70\% of patients. ${ }^{9}$ It has been suggested that four subtypes of PAN exist including: (1) Cutaneous PAN; (2) Systemic PAN; (3) Microscopic polyarteritis; and (4) Hepatitis B associated classic PAN.

Polyarteritis nodosum has been described in fewer than 250 children since the initial description by Kussmaul and Maier almost 140 years ago. ${ }^{3}$ The largest series by Ozen and colleagues contained $110 .{ }^{12}$ Systemic PAN was seen in $57.2 \%$, $30 \%$ had cutaneous PAN, $8.1 \%$ had microscopic PAN and $4.6 \%$ were associated with $\mathrm{HBs} \mathrm{Ag}$. Nervous system symptoms occurred in $14.5 \%$ of patients and were most common in those with systemic PAN. The specific neurological symptoms were not described, however, on imaging four patients had CNS artery aneurysms. In the systemic PAN group, remission occurred in $64 \%, 27 \%$ had persistent disease and $9 \%$ relapsed. The prognosis in juvenile PAN was better than in adults with a mortality of $1.1 \%$. A second series contained 31 patients. ${ }^{13}$ Peripheral nerve and CNS involvement was present in four (13\%) and nine (30\%) patients, respectively. CNS findings included: encephalitic symptoms $(n=4)$, persistent hemiparesis and ptosis $(n=1)$, hemiparesis and a seventh nerve palsy $(\mathrm{n}=1)$ and recurrent seizures $(n=3)$. The mortality rate in patients with nervous system involvement was $6.6 \% .{ }^{13}$ In a third series, 25 pediatric 


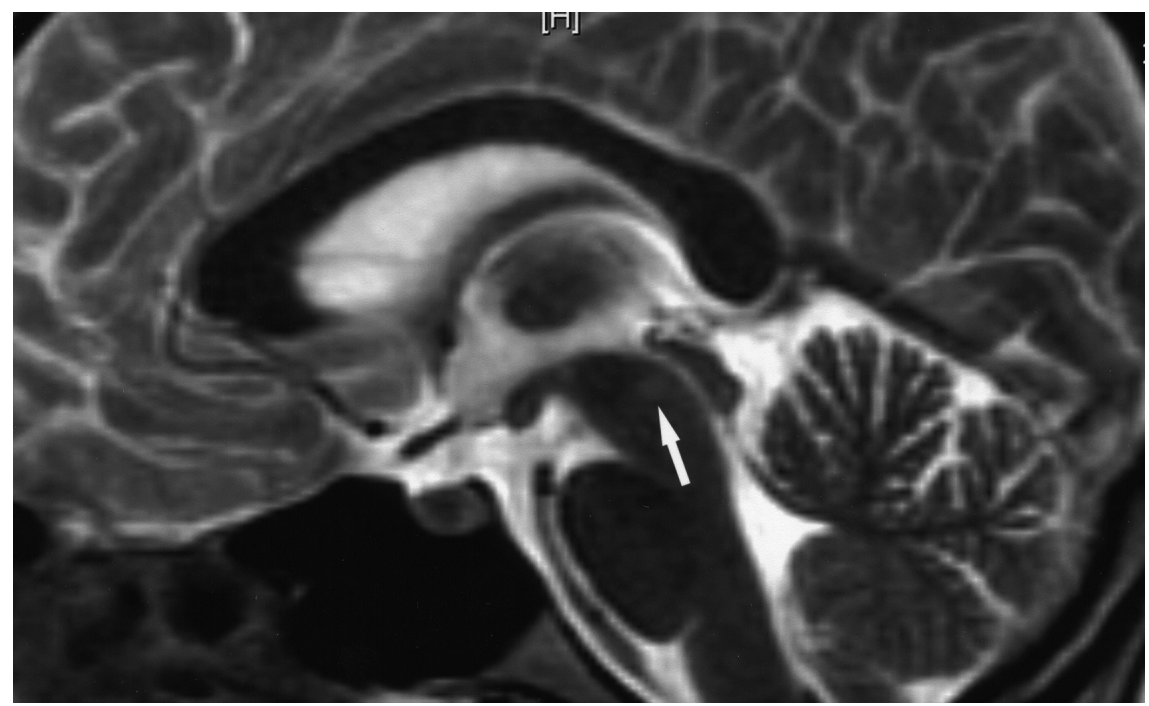

Figure 2. The MRI demonstrating infarct in the left paramedian midbrain. Sagittal T2 weighted image.

patients were studied and 56\% had neurologic involvement, but little clinical detail was provided. ${ }^{14}$ One case report describes a five year old girl with PAN who developed an ocular tilt reaction and left hemiparesis secondary to a right sided midbrain vasculitic lesion. ${ }^{15}$

A case report published by Schrodt and Callen (1999), suggests that vasculitis be considered among the toxicities associated with minocycline therapy. They reported on a 15 year old female who developed PAN after being treated with nine months of minocycline for acne vulgaris. ${ }^{16}$ Elkayam and colleagues in 1996, described minocycline-induced arthritis associated with fever, livedo reticularis and a positive p-ANCA in three women. The author noted rapid and complete resolution of symptoms after discontinuing the drug and recurrence after rechallenge. ${ }^{17}$ Neurological symptoms were not reported in any of the above cases. At the time of diagnosis, our patient was being treated with minocycline for acne which lends support to the above association. Additionally, her disease has come under good control since stopping the drug albeit with immune suppressive treatment.

The case demonstrates an unusual presentation of an uncommon disease. Convergence paralysis secondary to the vasculitis of PAN has not to our knowledge been described in a child. Our report raises further concern about the role of minocycline in PAN and other vasculitic syndromes.

\section{REFERENCES}

1. Lightfoot RW Jr, Michel BA, Bloch DA, Hunder GG, Zvaifler NJ, McShane DJ. The ACR 1990 criteria for the classification of PAN. Arthritis Rheum. 1990; 33: 1088-93.

2. Moore PM, Fauci AS. Neurologic manifestations of systemic vasculitis. A retrospective and prospective study of the clinicopathologic features and responses to therapy in 25 patients. Am J Med Sci. 1981; 71: 517-24.

3. Sundel R, Szer I. Vasculitis in Childhood. Rheum Dis Clin North Am. 2002; 28: 625-54.
4. Greenberg D, Aminoff M, Simon R. Disorders of equilibrium. In: Foltin J, Liebowitz H, Kurtz S, Panton N, editors. Clinical Neurology. New York: McGraw Hill; 2002. p. 115.

5. Brazis P, Lee A. Acquired Binocular Horizontal Diplopia. Mayo Clin Proc. 1999; 74: 907-16.

6. Ford RG, Siekert RG. Central nervous system manifestations of periarteritis nodosa. Neurology. 1965; 15: 114-22.

7. Guillevin L, Le THD, Godeau P, Jais P, Wechsler B. Clinical findings and prognosis of polyarteritis nodosa and Churg-Strauss angiitis: a study in 165 patients. Br J Rheumatol. 1988; 27: 258-64.

8. Provenzale J, Allen N. Neuroradiologic findings in Polyarteritis Nodosa. Am J Neuroradiol. 1996; 17: 1119-26.

9. Guillevin L, Fancois L, Gherardi, R. Polyarteritis Nodosa, Microscopic Polyangiitis, and Churg-Strauss syndrome: clinical aspects, neurologic manifestations and treatment. Neurol Clin. 1997; 15: 865-86.

10. Bouche P, Leger JM, Travers MA, Cathala HP, Castaigne P. Peripheral neuropathy in systemic vasculitis: clinical and electrophysiologic study of 22 patients. Neurology. 1986; 36: 1598-602.

11. Moore P, Richardson B. Neurology of the vasculitides and connective tissue diseases. J Neurol Neurosurg Psychiatry. 1998; 65: $10-22$

12. Ozen S, Anton J, Arisoy N, Bakkalodlu A, Besbas N, Brogan P, et al. Juvenile polyarteritis: results of a multicenter survey of 110 children. J Pediatr. 2004; 145:517-22.

13. Ozen S, Besbas N, Saatci U, Bakkaloglu A. Diagnostic criteria for Polyarteritis Nodosa in childhood. J Pediatr. 1992; 120: 206-9.

14. Fink C. Vasculitis. Pediatr Clin North Am. 1986; 33: 1203-19.

15. Ragge NK, Harris CM, Dillon MJ, Chong WK, Elston J, Taylor D. Ocular tilt reaction due to a mesencephalic lesion in juvenile Polyarteritis Nodosa. Am J Ophthalmol. 2003; 135: 249-51.

16. Schrodt B, Callen J. Polyarteritis Nodosa attributable to minocycline treatment for acne vulgaris. Pediatrics. 1999; 103: 503-4.

17. Elkayam O, Yaron M, Caspi D. Minocycline induced arthritis associated with fever, livedo reticularis, and p-ANCA. Ann Rheum Dis. 1996; 55: 769-71. 